\title{
Hacia una convergencia entre las tecnologías emergentes y las pedagogías emergentes
}

\author{
Sandra Milena Morales Mantilla ${ }^{1}$ \\ Pablo Munévar García ${ }^{2}$
}

\section{Resumen}

El presente artículo plantea una reflexión sobre la convergencia entre las tecnologías y las pedagogías emergentes, desde un análisis contemporáneo y actual en el modo de comprender el aporte de estas tendencias en la actual sociedad del conocimiento.

Palabras clave: pedagogías emergentes, tecnologías emergentes, sociedad del conocimiento, convergencia digital, didácticas digitales. 


\title{
Towards a convergence of emerging technologies and emerging pedagogies
}

\begin{abstract}
This article reflects on the convergence of technologies and pedagogies emerging from a contemporary and current analysis in the way of understanding the contribution of these trends in the current knowledge society.
\end{abstract}

Keywords: Emerging pedagogies, emerging technologies, knowledge society, digital convergence, digital teaching.

Recibido: 24/07/2014

Aceptado: 25/10/2014

\section{Introducción}

Actualmente los escenarios de formación en lo virtual se encuentran sometidos a una evolución producida no sólo por la incorporación de herramientas y recursos de lo tecnológico, sino también producto de los nuevos roles que los actores en cuanto a lo pedagógico se están configurando en dichos espacios. Esto se ratifica en posturas como la de Bauman (2008), quien en su teoría de las identidades líquidas analiza la manera en la cual la información «fluye», modificando formas de actuar y pensar cuando circula en el pensamiento y en los procesos neuronales, frente a las redes y la construcción de conocimiento de orden creativo. Es decir existen nuevas formas de «conocer el conocimiento» y por tanto nuevos roles que deben asumirse frente al tránsito de las sociedades del conocimiento a las sociedades del aprendizaje.

Esto se resignifica en la actualidad, ya que se puede decir que los avances tecnológicos van a la par con los pedagógicos (Facundo, 2008: 154), por este motivo la razón de ser y pensar en la generación de modelos que propicien el abordaje de orden histórico, crítico, reflexivo acerca de las tecnologías y su forma de ser incorporadas en la escuela; deben estar fundamentados a partir de procesos de enseñanza y aprendizaje basados en nuevos enfoques y nuevas maneras de orientar la formación de docentes en la actual sociedad del conocimiento.

La tecnología impregna casi todas las facetas imaginables de nuestra vida y actividades. El advenimiento de las tecnologías trajo cambios significativos en los últimos 20 años, afectando la cultura, la educación y la economía entre 
otros aspectos. Y mucho más este cambio se hace notable en la relación entre la interacción humana y la computadora. Esta relación, modificó la forma de construir conocimiento y la pedagogía tradicional en espacios digitales, cambiándolos de modos únicos (monomodos) en multimodales, lo monocultural en la multiculturalidad y lo analógico en lo digital, entre otros. Esta pedagogía digital ha surgido de forma concomitante con la producción de conocimiento del siglo XXI y la configuración de nuevos sujetos como ciudadanos globales en red (internautas) los cuales participan en la creación, el intercambio, el consumo y el mantenimiento de las fronteras culturales y espacio-temporales. En cierto modo, la actual aldea global se ha convertido en un espacio didáctico de cooperación basada en numerosos tipos de comunidades de conocimiento en red, que en conjunto crean y / o construyen conocimiento utilizando las redes sociales mediadas por tecnología.

Gracias a la mediación por diversas tecnologías digitales, «los internautas son cada vez multialfabetizados a través de contextos culturales, lingüísticos, científicos y geopolíticos» (Limbu, \& Binod, 2014: 14). A través de la comunicación en red, los internautas saben cómo otros ciudadanos del mundo están creando nuevas epistemologías, cómo están estableciendo dominaciones políticas y culturales, y la forma en que se están educando a nivel local, monitoreando contextos siempre cambiantes. De este modo, los internautas están globalizando sus historias y narrativas culturales, académicas y políticas. Como resultado de estos conocimientos locales con la apropiación de los procesos globales, la pedagogía del siglo XXI es potencialmente más difícil de alcanzar que las pedagogías pasadas (tradicionales).

La pedagogía del siglo XXI, en el advenimiento de la tecnología, modifica los paisajes de las pedagogías tradicionales, incluyendo la forma en que enseñamos, aprendemos y diseñamos currículos. Sobre todo con la aparición de nuevas tecnologías, como las redes sociales y la Web participativa (por ejemplo, la Web 2.0), los nuevos tipos de pedagogías han sido diseñados y desarrollados. Por ejemplo, los cursos abiertos masivos en línea (MOOC), el aprendizaje basado en el juego, la enseñanza virtual y aulas inmersivas, la simulación, el aprendizaje basado en escenarios, entre otros. Estas nuevas tecnologías también han generado la aparición de nuevos tipos de comunidades de aprendizaje electrónico (e-learning). A su vez, estas comunidades de aprendizaje electrónico han cambiado la naturaleza fundamental de aprendizaje y construcción del conocimiento. Más recientemente, la combinación de las características de la sociedad del conocimiento y de las sociedades red, las florecientes comunidades de conocimiento se han convertido en redes globales, instantáneas y transculturales. El desarrollo de la noción de vanguardia de las pedagogías emergentes, replantea la enseñanza, el aprendizaje, la didáctica y la construcción de la epistemología como fenómenos emergentes y multidimensionales que entrelazan el conocimiento intercultural, la tecnología, la pedagogía y la sociedad (por ejemplo, las sociedades, los conocimientos y la red misma) de manera compleja. 
Es por ello, que el concepto de tecnología emergente cobra vital relevancia, ya que en contraposición de la visión tecnocientífica y tecnócrata tradicional, se sustenta en el planteamiento de Veletsianos (2010), como:

Herramientas, conceptos, innovaciones y avances utilizados en diversos contextos educativos al servicio de diversos propósitos relacionados con la educación. Además, propongo que las tecnologías emergentes ("nuevas" y "viejas") son organismos en evolución que experimentan ciclos de sobreexpectación y, al tiempo que son potencialmente disruptivas, todavía no han sido completamente comprendidas ni tampoco suficientemente investigadas (Veletsianos 2010: 3-4).

Estos aspectos ratifican los planteamientos de Boaventura de Sousa Santos (2001), sobre la pertinencia de abordar esas nuevas formas de comprender la acción pedagógica desde las diversas subjetividades en la cultura escolar. La educación deja de ser un problema eminentemente escolar, ya hace parte de la cultura misma en cualquier escenario de la sociedad y la comunidad, debido a que las formas de aprender las encontramos no solo asequibles en aulas sino en cualquier sitio, gracias a las tecnologías.

Ahora, el concepto que permite relacionar lo concerniente a las tecnologías emergentes con las pedagogías emergentes lo podemos encontrar en Adell y Castañeda quienes las proponen como «el conjunto de enfoques e ideas pedagógicas, todavía no bien sistematizadas, que surgen alrededor del uso de las TIC en educación y que intentan aprovechar todo su potencial comunicativo, informacional, colaborativo, interactivo, creativo e innovador en el marco de una nueva cultura del aprendizaje» (2012: 3).

Con la evolución social -aunque siempre con retraso- la escuela tradicional ha ido dejando en el camino los aspectos más externos que simbolizaban «lo tradicional como obsoleto» (Trilla, 1996:17), determinadas costumbres como el castigo físico, los modales rancios y desfasados, los métodos de enseñanza acientíficos basados en el mero verbalismo y la repetición, los libros con contenidos demasiado anticuados con respecto al desarrollo científico, el mobiliario arcaico y el ambiente arquitectónico disfuncional... y, por supuesto, los antiguos planes de estudio; y ha ido adecuándose, también externamente, a los nuevos requerimientos de las sociedades industriales avanzadas, cambiando el currículum, los edificios, los libros de texto, etc., pero sin modificar sus verdaderas funciones básicas, sin replantear a fondo su finalidad y -coherentemente- sin cambiar tampoco en profundidad la formación de los docentes. Se ha buscado, por tanto, una escuela más moderna, más técnica (Gimeno Sacristán, 1982), pero igualmente garante de la función de reproducción social que la sociedad, supuestamente, espera de ella.

En este sentido se resalta el aporte pedagógico y didáctico en tendencias alternativas como la mirada de los modelos didácticos racional-tecnológicos, los 
cuales enfatiza García Pérez (2000). Se define en la búsqueda de una formación más «moderna» para el estudiantado -entendida, en cualquier caso, como formación cultural, no como desarrollo personal- conlleva la incorporación a los contenidos escolares de aportaciones más recientes de corrientes científicas, o incluso de algunos conocimientos no estrictamente disciplinares, más vinculados a problemas sociales y ambientales de actualidad. Así mismo, se insertan -más que integrarse- en la manera de enseñar determinadas estrategias metodológicas (o técnicas concretas) procedentes de las disciplinas. Se suele depositar, a este respecto, una excesiva confianza en que la aplicación de esos métodos va a producir en el alumno el aprendizaje de aquellas conclusiones ya previamente elaboradas por los científicos. Para ello se recurre a la combinación de exposición y ejercicios prácticos específicos, lo que suele plasmarse en una secuencia de actividades, muy detallada y dirigida por el profesor, que responde a procesos de elaboración del conocimiento previamente determinados (en cuanto que es un camino ya recorrido por la ciencia de referencia) y que puede incluso partir de las concepciones de los alumnos con la pretensión de sustituirlas por otras más acordes con el conocimiento científico que se persigue. Sin embargo, junto con este «directivismo» encontramos, a veces, otra perspectiva en la que la metodología se centra en la actividad del alumno, con tareas muy abiertas y poco programadas que el profesor concibe como una cierta reproducción del proceso de investigación científica protagonizado directamente por dicho alumno. Se da así una curiosa mezcla de contenidos disciplinares y metodologías «activas», que, por encima de su carácter «dual» (es decir, esa mezcla de tradición disciplinar y de activismo), encuentra cierta coherencia en su aplicación, satisfaciendo por lo demás diversas expectativas del profesorado y de la sociedad. A la hora de la evaluación se intenta medir las adquisiciones disciplinares de los alumnos, aunque también hay una preocupación por comprobar la adquisición de otros aprendizajes más relacionados con los procesos metodológicos empleados.

Existen otras funciones que se pueden asignar a las tecnologías emergentes presentes en los medios de comunicación (Bautista y Jiménez, 1991); concretamente podemos contemplarlos como herramientas para resolver problemas en diferentes ámbitos, o la de ser soportes de sistemas de representación que podemos utilizar para expresar afectos, sentimientos, o cualquiera de las sensaciones o contradicciones internas que caracterizan a los humanos. También, entre otras funciones emergentes que se pueden asociar a los recursos está la de permitir analizar el contenido de los discursos realizados por representantes de los órganos del poder, así como permitir a un grupo de profesores reflexionar y deliberar sobre sus ámbitos de trabajo con el fin de detectar dificultades en los alumnos, desigualdades, injusticias (...) o cualquiera de las miserias presentes en la sociedad actual. Es decir, entre las nuevas funciones no sólo está resolver problemas sino, también, diagnosticarlos y plantearlos.

Esto se evidencia actualmente en posturas emergentes como los planteamientos de Siemens (2010), que basado en los distintos paradigmas que rigen la interacción 
en la red, son consecuentes con las directrices propias de la relación entre hombre - máquina. Es decir teorías como el conectivismo, permiten fomentar o animar el debate académico en torno al comportamiento del individuo en lo relacionado con las interacciones formativas mediadas por tecnologías.

Desde la perspectiva de los ambientes de aprendizaje, los profesores más innovadores apropian la tecnología para transformar su práctica docente y la manera cómo sus estudiantes aprenden. Aunque como señala Adell (1997), los primeros usos de esta tecnología fueron para replicar las prácticas presenciales tradicionales, es decir modelos basados en la transmisión de conocimiento. Sin embargo, al ir ampliándose el uso de estos recursos tecnológicos y las experiencias que incorporan nuevos enfoques metodológicos, se ha ido contando con una mayor variedad de uso de la Web en la educación.

Con estas condiciones, que se encuentran en las plataformas virtuales basadas en los sistemas LMS que son evidencias del constructivismo social, la estructura de lo que se refiere a los modelos de formación se fundamentan en enfoque metodológico e-learning. Este enfoque supera en valor instrumental el uso de las herramientas 2.0 (Muras, 2008: 7) y asegura las condiciones de una web 2.0 que desarrolla nuevas maneras de innovar y de gestionar estrategias de enseñanzaaprendizaje desde las TIC. Es decir que son «aquellas formas de aprendizaje asistidas o apoyadas por medios electrónicos que se aplican en el ámbito de la formación y perfeccionamiento profesionales» (Schüpbach et al., 2003: 9).

El e-learning, el blended-learning, m-learning como ejemplos de éstos enfoques metodológicos, contienen en sí mismos matices y particularidades diferenciadoras en relación con los métodos y propósitos de aprendizaje mediante el uso de medios electrónicos (Dorado Perea \& Rodríguez, 2004: 4). En el argot pedagógico actual, dichos enfoques metodológicos se relacionan con las denominadas «modalidades» en las cuales se desarrolla la propuesta curricular en lo virtual. El enfoque e-learning se encuentra estructurado en dos grandes sistemas que caracterizan su implementación en los AVA: los WBT o CW y los LMS/CMS. Según Bartolomé y Grané (2010) el primero que corresponde a los denominados WBT (Web Based Traning) o CW (Course Ware) los cuales son los contenidos adaptados didácticamente de los materiales educativos en diversos formatos multimedia y los segundos LMS (Learning Managment System) / CMS (Content Management SystemWeb Based Training), que son plataformas o sistemas de gestión de contenidos para el aprendizaje como herramientas informáticas que permiten la visualización y uso de los contenidos y la gestión de conocimiento entre los participantes, alumnos y tutores.

Autores como Sangrá y Wheeller (2013), sitúan la importancia de la emergencia de las iniciativas de contenido generado por el usuario frente a lo tecnológico, en las que el contenido no se entrega a los estudiantes sino que lo crean ellos mismos conjuntamente: 
El aumento de las prácticas educativas abiertas (OEP) y los cursos abiertos en línea y masivos (MOOC) y la creación de nuevos proveedores de soluciones de autoaprendizaje como OER University, Peer2Peer University o University of the People, están transformando escenarios conocidos en otros dominios de una naturaleza mucho más incierta. Esta tendencia plantea un reto para las instituciones conservadoras, especialmente las universidades. El aprendizaje es cada vez más informal a medida que las personas desarrollan redes complejas para ayudarse entre sí, de modo que intensifican la percepción de que la enseñanza y la formación ya no están proporcionadas exclusivamente por las instituciones, sino que ambas pueden también resultar de una colaboración entre individuos y sus redes especializadas. Mientras que algunas universidades muy reconocidas como MIT o Harvard han firmado un acuerdo con Coursera, una compañía que proporciona una plataforma para distribuir MOOC a cualquier persona en cualquier sitio, mediante un enfoque conductista tradicional (Bates, 2012), otras han fundado nuevas organizaciones para proporcionar aprendizaje, como es el caso de Udacity (p. 110).

Gracias a la manera en la cual la sociedad de la información ha trascendido a la sociedad del conocimiento, es decir el paso de una mirada capitalista de acumulación de información - desde lo que se denominaba en su momento el saber sabio - a una mirada más del saber contextualizado - producto de los procesos de construcción del conocimiento -, la didáctica se ha posicionado como una ciencia que realmente le ha aportado significancia a los nuevos enfoques pedagógicos, principalmente aquellos que han surgido en la apropiación de las TIC en el escenario educativo.

Para Picardo Joao (2001), la didáctica toma un papel preponderante en la articulación entre lo tecnológico y lo pedagógico desde lo emergente, en lo que él denomina pedagogía informacional ya que hace alusión a todos los recursos asociados a la información; entre ellos se destacan: internet, medios de comunicación, libros, CD-ROM, y otros datos estadísticos y significativos que están en el entorno aportando algún indicio informacional; no obstante, no es novedoso que esto sea información, lo que sí cambia, es el lugar de estos referentes, ya que la pedagogía informacional exige que estos recursos ingresen al aula, y se tornen una plataforma para el proceso de enseñanza aprendizaje.

La información, desde el punto de vista didáctico, son los datos tangibles e intangibles que posibilitan nuevos conocimientos, o bien, la base sobre la cual se piensan, discuten, analizan y proponen ideas y proyectos. Dicho de otro modo, ya no basta que exista un libro de texto y un retroproyector de acetatos para desarrollar una clase, ahora se necesitan: a) los diversos partes noticiosos para discutir la problemática social, cultural, económica y política; b) los motores de búsqueda para acceder a información pertinente y a antecedentes sobre el tópico estudiado; c) el correo electrónico para comunicarse con más eficiencia; d) la creación de redes de trabajo para 
optimizar los grandes volúmenes de información; e) los espacios virtuales o digitales para registrar o discutir la información; y f) la creación de una nueva cultura académica sustentada en el aprendizaje permanente; entre otras formas o expresiones didácticas (Picardo Joao, 2001: 29).

La introducción de los entornos virtuales de enseñanza - aprendizaje en cualquier escenario de formación es ya una realidad. En este momento se puede afirmar que la mayoría de las instituciones de educación superior presentan, con mayor o menor acierto, esos entornos virtuales de enseñanza - aprendizaje tanto como un elemento clave en sus estudios, que como muestra de su capacidad de innovación. Ahora bien, no siempre avanza de forma paralela la apuesta de las universidades por la introducción de las tecnologías de la información y la comunicación en sus aulas, con la formación de su profesorado en estas mismas herramientas. Por lo que, si queremos acometer estos procesos de innovación, será necesario que las instituciones universitarias apuesten por la formación de su profesorado, pero desde una perspectiva crítico pedagógica, rechazando la visión instrumental de la tecnología como un fin en sí misma, o simplemente como una moda didáctica más.

Para Bautista, Borges \& Forés (2006), todo entorno virtual de aprendizaje, exige «una planificación y un desarrollo didáctico diferente. Las estrategias de aprendizaje, los procesos de comunicación, de evaluación, etc. no se desarrollan de la misma forma, por lo que se precisa de una formación específica que enseñe a abordar de la mejor manera posible ese proceso educativo» (p. 96). Se reconoce la importancia de la experiencia del docente en los escenarios presenciales, pero se debe aclarar que, en cuanto se accede al aula virtual, estamos en otro espacio de aprendizaje que sigue sus propias reglas, debido a que existen aspectos de orden sistemático, didáctico y metodológico de cómo debe llevarse a cabo un proceso de enseñanza - aprendizaje universitario en un entorno virtual.

Bautista, Borges \& Forés (2006), señalan que «desde el ámbito universitario y para el profesorado universitario, el desempeño docente mediado por entornos virtuales y quizás a desaprender, reaprender o analizar en profundidad algunas de las estrategias y prácticas educativas que hemos entendido como útiles y eficaces para estos entornos» (p. 15). Teniendo claro que aunque hablemos de virtualidad, en estos escenarios lo único virtual es el contexto. El profesorado, los estudiantes, el aprendizaje... son reales y como tal debemos atenderlo.

Un docente en un entorno virtual exige preparación, actuaciones y actitudes distintas, que, en definitiva, se concretan en saber acompañar a través de la red a cada estudiante a lo largo de su aprendizaje. Esta es la función clave del docente en estos escenarios, sin dejar de lado las otras funciones implícitas en toda tarea docente. 
Para poder llevar a cabo esta función de acompañamiento es imprescindible el diseño y la planificación de todo el proceso de enseñanza - aprendizaje: el diseño de toda acción formativa, la acción del docente en cada momento, el seguimiento y la comunicación a lo largo de todo el proceso, los materiales y los recursos didácticos para la formación en línea, la evaluación continua como clave de este seguimiento, etc. En cada uno de estos puntos, no sólo se expone cómo debe desempeñarse, sino que se evidencian también los problemas que pueden surgir, los errores más comunes que se cometen, lo que se espera del profesor en cada fase. Resulta muy ilustrativa la inclusión al final de cada capítulo de un apartado en el que se recoge la voz de docentes y estudiantes, exponiendo su experiencia en cada una de estas fases. Lo que ha valorado, los aciertos y los errores.

En estos nuevos entornos el papel del profesor será notablemente diferente al que normalmente desempeña en la formación tradicional-presencial, de forma que de la función del profesor como transmisor de información pasará a desempeñar otras más significativas, como la de diseñador de situaciones mediadas de aprendizaje, tutor y orientador virtual, diseñador de medios, etc. No podemos dejar de reconocer que, de todas estas funciones, la de tutoría virtual es de máxima importancia y relevancia para garantizar una acción educativa de calidad y que, como señalamos en otro lugar (Cabero, 2006), desempeñarán diferentes funciones que superan la académica (técnica, orientadora, organizativa y social.

Un Entorno Virtual de Aprendizaje, por tanto, permite distribuir materiales educativos en formato digital (textos, imágenes, audio, simulaciones, juegos, etc.), realizar discusiones en línea, integrar contenidos relevantes de la red o para posibilitar la participación de expertos o profesionales externos en los debates o charlas. En un EVE/A se combinan herramientas: para la comunicación síncrona y asíncrona; para la gestión de los materiales de aprendizaje; para la gestión de las personas participantes, incluidos sistemas de seguimiento y evaluación del progreso de los estudiantes. Desde el punto de vista didáctico, un EVE/A ofrece soporte tecnológico a profesores y estudiantes para optimizar distintas fases del proceso de enseñanza/aprendizaje: planificación, implementación, desarrollo y evaluación del currículum. Un EVA trata de aprovechar, en beneficio de la educación, el espacio que la informática y las telecomunicaciones hacen posible (Duart y Sangrà, 2007).

Es decir, un Ambiente Virtual de Aprendizaje es el conjunto de entornos de interacción, sincrónica y asincrónica, donde, con base en un programa curricular, se lleva a cabo el proceso enseñanza-aprendizaje, a través de un sistema de administración de aprendizaje (López et al., 2002).

La idea que subyace a los AVA, es decir los PLE (Personal Learning Environment) no es nueva. Brown (2010), sitúa su origen en 2001, el año del inicio del proyecto NIMLE (Northern Ireland Integrated Managed Learning Environment). NIMLE 
fue el primer proyecto financiado por el JISC (el Joint Information Systems Committee de la Gran Bretaña) en el que se concebía un entorno de aprendizaje centrado en un alumno que podría moverse entre varias instituciones educativas y administrar las fuentes de información de cada una de ellas.

Sin embargo, el acrónimo PLE no apareció hasta noviembre de 2004 (Severance, Hardin \& Whyte, 2008), como parte del título de una de las sesiones de la JIS C/CETIS Conference de aquel año. Al año siguiente, el Centre for Educational Technology and Interoperability Standards (CETIS) recibió fondos del JISC para desarrollar una especificación de estándares para PLE y crear un modelo de referencia con un prototipo de software libre que permitiera su implementación como tecnología. Las herramientas PLEW (el servidor) y PLEX (la aplicación de escritorio) se desarrollaron en este proyecto (CETIS, 2006).

Por tanto, un PLE se concibe como el conjunto de herramientas, fuentes de información, conexiones y actividades que cada persona utiliza de forma asidua para aprender. Es decir, que el entorno personal de aprendizaje incluye tanto aquello que una persona consulta para informarse, las relaciones que establece con dicha información y entre esa información y otras que consulta; así como las personas que le sirven de referencia, las conexiones entre dichas personas y él mismo, y las relaciones entre dichas personas y otros que a la larga pueden resultarle de interés; $y$, por supuesto, los mecanismos que le sirven para reelaborar la información y reconstruirla como conocimiento, tanto en la fase de reflexión y recreación individual, como en la fase en la que se ayuda de la reflexión de otros para dicha reconstrucción.

Así mismo, enmarcados en el ámbito tecnológico en el que nos desenvolvemos - la Internet social - , consideramos que dicho entorno se explicita en el conjunto de herramientas que canalizan dichas fuentes, actividades y conexiones. En consecuencia, el PLE se ve condicionado por dichas herramientas en la medida en que determinan la forma en la que accedemos a ellas, las utilizamos y combinamos.

Los PLE van mucho más allá de las tecnologías instrumentales, ya que implican incluso aquellos espacios y estrategias del mundo presencial, que la persona utiliza para aprender. El término PLE alude a la parte de ese entorno que se nutre y amplía en el marco de la tecnología (no de una en particular, sino de todas las TIC en general).

En conclusión, el tránsito de los AVA a los PLE pasando por los EVA, supone una nueva forma de asumir la acción tutorial ya que los aspectos didácticos, los propios de las teorías del aprendizaje, los aspectos didácticos y por supuesto de diseño de estos escenarios, buscan resignificar el papel de la formación. Esto es importante para la educación en la virtualidad, ya que mientras el Ambiente Virtual de Aprendizaje se caracterizaba por el conjunto de recursos, actividades, 
metodologías y estrategias de evaluación, los Entornos Virtuales de Aprendizaje se fundamentan en una postura ecológica del aprendizaje que está por encima de los mismos AVA, son escenarios de construcción de conocimiento en el cual está definido no por un ambiente solamente sino por varios entornos. El carácter ecológico se da por la diversidad de entornos los cuales potencian la acción tutorial, acudiendo a lo didáctico en pro de ser un entorno tecnológicamente enriquecido para aprender, que contiene y sustenta elementos integrados que median la actividad como un sistema íntegro de actuación para el aprendizaje, es decir, como un sistema complejo de acción tutorial, no sólo como operaciones específicas, sino con un contundente efecto mediacional de un sistema de acción tutorial en la construcción del conocimiento. «Como en el aprendizaje, es mayor cuando: el sistema de actuación es más complejo y cuando se usa de manera exclusiva» (Suárez Guerrero, 2003: 3).

\section{Conclusiones}

Los estudios frente a la formación en los entornos virtuales, como los liderados por Tomoko Hamada (2003), concluyen que la tecnología nos está forzando a examinar los papeles que juegan las universidades en la creación y propagación de la sabiduría humana. Es decir se configuran nuevas formas de enseñar y aprender, modelos de formación y el uso al máximo de recursos educativos con el fin de acceder a la sabiduría y el conocimiento que se propaga por las redes de información.

Al respecto Fernández (2006), menciona lo siguiente:

Los entornos virtuales pretenden crear un aula donde ésta no está disponible, o bien ampliar las potencialidades del aula real. Los entornos virtuales de aprendizaje son eso, virtuales: reproducen el modelo de enseñanza/ aprendizaje que tiene el profesor. Si su modelo es transmisor en el aula, en su virtualidad electrónica también lo será (p. 3).

La UNESCO (2014), en su informe Global de Educación señala que el entorno virtual de aprendizaje es una forma totalmente nueva de Tecnología Educativa y ofrece una compleja serie de oportunidades y trabajo en las instituciones educativas en todo el mundo. Un entorno virtual de aprendizaje se define como un programa informático interactivo de carácter educativo que integra la comunicación, que significa que está asociado con las nuevas tecnologías. Mientras que las tecnologías están en desarrollo, las nuevas formas de trabajo y la interacción entre los usuarios se crean. El uso educativo de estas nuevas formas se refleja en proyectos de vanguardia que crean entornos de aprendizaje innovadores y nuevas experiencias de aprendizaje. Estas redes o redes electrónicas, compuesto por personas con comunes intereses, abren posibilidades para la enseñanza y el aprendizaje donde las personas involucradas se identifican en termino de propios y comunes objetivos que motivan su participación. 
Ahora, la nueva idea de un Entorno Personal de Aprendizaje (PLE) reconoce que el aprendizaje es continuo y busca brindar herramientas de apoyo al aprendizaje mismo. También reconoce el papel del individuo en la organización de su propio aprendizaje, es decir en la educación en la virtualidad la acción tutorial en estos entornos no recae en la figura exclusiva del tutor. Un PLE se basa en la idea de que el aprendizaje se lleva a cabo en diferentes contextos y situaciones y no será proporcionado por un proveedor de aprendizaje (tutor), sino por el individuo que diseña sus propias formas de aprender.

Los PLE proporcionan a los estudiantes desarrollar propios espacios bajo su propio control para desarrollar y compartir sus ideas. Además de proporcionar un enfoque más holístico a los ya masificados ambientes de aprendizaje, ya que reúne a las fuentes y los contextos de aprendizaje hasta ahora separados. Los estudiantes aprenden a asumir la responsabilidad de su propio aprendizaje con una acción tutorial de carácter autónoma. Fundamentalmente, los PLE puede cerrar los jardines amurallados de las instituciones educativas con los mundos exteriores. De este modo los alumnos pueden desarrollar los juicios y las habilidades de comprensión o la innovación mediada por las nuevas tecnologías buscando transformar y cambiar la sociedad.

Desde la acción tutorial los Entornos Personales de Aprendizaje no son una simple aplicación, sino más bien un nuevo enfoque para la apropiación de las nuevas tecnologías para el aprendizaje. La construcción de entornos personales del aprendizaje no es técnica, sino más bien una forma de que se legitima desde lo filosófico, ético y pedagógico hacia una real educación en la virtualidad.

Una convergencia real entre tecnologías y pedagogías emergentes, solo es posible si existe un reconocimiento que la apropiación del saber tecnológico por encima del valor de uso o el instrumental, que muchas veces se encuentra en los procesos formativos en los entornos apoyados en virtualidad. La resignificación del valor histórico, crítico y hermenéutico que ha aportado la tecnología a los procesos pedagógicos, son los que permiten legitimar que aquellos procesos que emergen del desarrollo de estrategias que son los que permiten potenciar realmente el aprendizaje.

No basta con un conjunto de recursos, herramientas, plataformas y contenidos, si ellos no tienen un sentido, si no son significativos y si no permiten que el conocimiento pueda «emerger» y pueda evolucionar, ya que desde estas formas de pedagogías, son las que realmente aportan a la tecnología en su establecimiento como saber contemporáneo apropiado en la educación. 


\section{Referencias bibliográficas}

Adell, J. 1997. Tendencias en educación en la sociedad de las tecnologías de la información, EDUTEC. Revista Electrónica de Tecnología Educativa, № 7. Disponible en http://www.uib.es/depart/gte/revelec7.html. Consultado en enero de 2014.

Adell, J. y Castañeda, L. 2012. Tecnologías emergentes, ¿Pedagogías emergentes? En J. Hernández, M. Pennesi, D. Sobrino y A. Vázquez (coords.). Tendencias emergentes en educación con TIC. Pp. 13-32. Barcelona: Asociación Espiral, Educación y Tecnología.

Bartolomé, A. y Grané, M. 2010. "Herramientas digitales en una Web ampliada”. En de Pablos J. (Coord.). Tecnología Educativa. La formación del profesorado en la era de Internet. Málaga: Aljibe.

Bauman, Z. 2008. Los retos de la educación en la modernidad líquida. Barcelona: Gedisa.

Bautista, G., Borges, F. y Forés, A. 2006. Didáctica universitaria en entornos virtuales. Madrid: Narcea.

Bautista, A. \& Jiménez Benedit, M. 1991. Uso, selección de medios y conocimiento práctico del profesor, Revista de Educación, 296, pp. 299-326.

Brown, S. 2010. From VLEs to learning webs: the implications of Web 2.0 for learning and teaching. Interactive Learning Environments 18(1), 1-10

Cabero, J. 2006. Bases pedagógicas del e-learning. Revista de Universidad y Sociedad del Conocimiento (RUSC) Vol. 3 (1). Disponible en: http://www.uoc.edu/rusc/3/1/dt/ esp/cabero.pdf. Consultado en abril de 2011.

CETIS. 2006. The personal learning environments reference model project. JISC CETIS Wiki. Recuperado en marzo de 2014, del sitio http://wiki.cetis.ac.uk/Ple .

De Kerckhove, D. 1999. Inteligencias en conexión. Hacia una sociedad de la web. Barcelona: Gedisa.

Dorado, C. \& Rodríguez, D. 2004. El trabajo en red como fuente de aprendizaje: posibilidades y límites para la creación de conocimiento. Una visión crítica Revista Educar. No 28: 11-24. Disponible en: http://ddd.uab.es/pub/educar/0211819Xn37p11.pdf Consultado en mayo de 2012.

Duart, J. \& Sangra, A. 2007. Aprender en la virtualidad. Barcelona: Gedisa

Facundo, A. 2008. El marco regulatorio de la educación a distancia en América Latina y el Caribe. Bogotá: Virtual Educa, UNAD. 
Fernández, J. 2006. La plataforma educativa. La hora del e-aprendizaje. Educación Linux. User. Número 13. Disponible en: http://linux-magazine.es. Consultado en mayo de 2013

García Pérez, F. 2000. Los modelos didácticos como instrumentos de análisis y de intervención en la realidad educativa. Revista bibliográfica de Geografía y Ciencias Sociales, № 207, 18 de febrero de 2000. Disponible en http://www.ub.es/geocrit/b3w207.htm Consultado en diciembre de 2013.

Gimeno, J. 1982. La pedagogía por objetivos: obsesión por la eficiencia. Madrid, Morata.

Hamada, T. 2003. Tecnología informática y educación: perspectiva teórica. En: C. Bueno y M.J. Santos (Coord.s). Nuevas Tecnologías y cultura. Pp. 183-208. Barcelona: Anthropos Editorial.

Lévy, P. 2004. Inteligencia colectiva: por una antropología del ciberespacio. Disponible en: http://inteligenciacolectiva.bvsalud.org/public/documents/pdf/es/ inteligenciaColectiva.pdf. Consultado en noviembre de 2012.

Limbu, M. \& Binod, G. 2014. Emerging Pedagogies in the Networked Knowledge Society: Practices Integrating Social Media and Globalization. Disponible en: http://www.igiglobal.com/pdf.aspx?tid=96047\&ptid=78256\&ctid=15\&t=foreword. Consultado en abril de 2014 .

López, E. \& Ledesma. 2002. Ambientes Virtuales De Aprendizaje, Instituto Politécnico Nacional, Presimposio Virtual SOMECE. Memorias disponibles en http://www. somece.org.mx/virtual2002. Consultado en Diciembre de 2013

Moreno, O. \& Sola, M. 2005. La acción tutorial en el contexto del espacio europeo de educación superior. En: Revista Educación y Educadores, № 8: 123-143.

Munévar, P., Lasso, E. \& Rivera, J. 2013. Nuevas comprensiones de los roles del tutor y el estudiante en la educación en la virtualidad. En: Memorias XIV Encuentro Virtual Educa. Virtual Educa. Medellín: Disponible en http://www.virtualeduca.info/ ponencias2013/344/PonenciaRolesdeltutorUbuntuVirtualeduca.pdf. Consultado en octubre de 2014 .

Muras, M. 2008. Nuevas Tecnologías en el ámbito de la Educación. E-learning Más allá del 2.0. Disponible en http://es.slideshare.net/tumbukta/elearning-ms-all-del-20presentation-728611? related $=1$. Consultado en mayo de 2014.

Picardo, J. 2001. Espacios y tiempos de la educación. San Salvador: Servicios Educativos: 21- 42.

Sangrà, A. \& Wheeler, S. 2013. Nuevas formas de aprendizaje informales: ¿O estamos formalizando lo informal? Revista de Universidad y Sociedad del Conocimiento (RUSC). Vol. 10 (1): 107-115. 
Santos, B. 2001. Nuestra America: Reinventing a Subaltern Paradigm of Recognition and Redistribution, Theory, Culture and Society, 18, 2-3.

Severance, C., Hardin, J. \& Whyte, A. 2008. The coming functionality mash-up in personal Learning environments. Interactive Learning Environments, 16(1), 47-62.

Suárez, C. 2003. Los entornos virtuales de aprendizaje como instrumento de mediación. En Revista Teoría de la Educación. Universidad de Salamanca.

Schüpbach, E., Guggenbühl, U. y Krehl, C. 2003. Didaktischer Leitfaden für E- Learning. Berlín: Hep-Verlag.

Siemens, G. 2010. Conociendo el conocimiento. New York Press

Trilla, J. 1996. Tendencias educativas: Escuela tradicional. Pasado y presente. Cuadernos de Pedagogía, 1996, n² 253, p. 14-19.

UNESCO. 2014. Informe Global de Educación. Educación Para Todos (EPT). Disponible en: http://www.unesco.org/ept-2014.pdf . Consultado en marzo de 2014.

Veletsianos, G. 2011. Designing Opportunities for Transformation with Emerging Technologies. Educational Technology, 51(2): 41-46.

Veletsianos, G. 2010. A Definition of Emerging Technologies for Education. En: G. Veletsianos (ed.) Emerging Technologies in Distance Education. Pp. 3-22. Athabasca, CA: Athabasca University Press. 